\title{
Testing the Judgment-Related Account for the Extinction of Evaluative Conditioning
}

\author{
Tal Moran ${ }^{12}$, Tzipi Dror ${ }^{23}$, Yoav Bar-Anan ${ }^{3}$ \\ ${ }^{1}$ Department of Experimental Clinical and Health Psychology, Ghent University, \\ Belgium \\ ${ }^{2}$ Department of Psychology, Ben-Gurion University of the Negev, Israel \\ ${ }^{3}$ Department of Psychology, Tel-Aviv University, Israel
}

\section{In press, Cognition \& Emotion}

The data that support the findings of this study are openly available in http://doi.org/10.17605/OSF.IO/XUHPT.

Author Note:

Tal Moran and Tzipi Dror have equal contributions. Correspondence concerning this article should be addressed to Tal Moran, Department of Experimental Clinical and Health Psychology, Ghent University, Belgium, Email: Tal.MoranYorovich@UGent.be. This research was supported by a grant from the Israel Science Foundation [779/16] to Y. B.A. 


\begin{abstract}
Evaluative Conditioning (EC) effects refer to changes in the liking of a neutral (conditioned) stimulus (CS) due to pairing with an affective (unconditioned) stimulus (US). Some research found that EC effects are resistant to presentations of the CS without the US, whereas other studies found evidence for extinction effects. A recent study found extinction of EC only when participants rated the CS before and after the CS-only presentations, but not when CS evaluation was measured once or indirectly with the Evaluative Priming task. In two experiments (total $\mathrm{N}=$ 2,181), we found no evidence that indirectly measured evaluation is sensitive to extinction, using an indirect evaluation measure with high sensitivity - the Implicit Association Test. However, unlike previous research, we found that evaluation of any stimuli (and not only the CS) before the CS-only presentations decreases self-reported EC effects. Our results are compatible with the conclusion that the extinction of EC is limited to evaluation measured directly. We discuss the theoretical implications of these results, and conclude that the specific conditions (and mechanisms) that change the direct evaluative response are yet to be clarified.
\end{abstract}

Key words: Evaluative Conditioning; Extinction; Evaluative judgement 


\section{Testing the Judgment-Related Account for the Extinction of Evaluative Conditioning}

To sell a new product, marketing ads usually present the product together with positive objects like puppies, smiling children or beloved celebrities. The logic behind this strategy is that the pairing of the new product with positive objects will result in a positive evaluation of the new product. Indeed, extensive research has found that when a neutral conditioned stimulus (CS) repeatedly occurs with a positive or a negative unconditioned stimulus (US), the evaluation of the CS changes, usually toward the valence of the affective stimulus, an effect known as Evaluative Conditioning (EC; De Houwer, Thomas, \& Baeyens, 2001; Hofmann, et. al., 2010). But would the evaluation of the new product remain positive even after people no longer see it with the positive object, for example when encountering it repeatedly at the store? In other words, are EC effects resistant to extinction?

If EC effects are not resistant to extinction, then the effectiveness of the above-mentioned marketing strategy is questionable. On a theoretical level, knowing if EC effects are resistant to extinction or not informs and constrains models about the processes that underlie EC. Some existing theories predict resistance of EC effects to extinction while others predict extinction effects (see Hofmann et al., 2010, and Walther, Weil, \& Langer, 2011, for a discussion).

Early research has shown that EC is resistant to extinction: EC effects remained the same after repeated presentations of the CS alone without the US (e.g., Baeyens et al., 1988; Díaz, Ruiz, \& Baeyens, 2005; Hermans et al., 2002; Vansteenwegen et al., 2006). Later, however, this finding has been challenged. First, a meta-analysis of EC research (Hofmann et al., 2010) found that EC effects measured after presenting CS-US pairs (hereafter, post-pairing measurement) were larger than EC effects measured after the pairing was followed by CS-only presentations 
(hereafter, post-CS-only measurement) ${ }^{1}$, suggesting extinction of EC effects. Second, research found that the rating of the CS post-pairing and prior to CS-only presentations is an important factor for determining whether EC effects would seem resistant to extinction or not. Specifically, an experiment that tests extinction of EC usually includes two phases. In the first phase, participants observe CS-US pairing trials. In the second phase, participants observe CS-only trials. To test if an extinction effect occurred, the evaluation of the CS measured post-pairing is compared with the CS evaluation measured post-CS-only. These two evaluations can be measured between-participants (i.e., one group of participants evaluates the CS post-pairing, and a second group evaluates the CS post-CS-only), or within-participants (i.e., all participants provide the two evaluations). Previous research found extinction effects only in withinparticipants designs, when the same participants rated the CS both post-pairing and post-CS-only (Gawronski, Gast, \& De Houwer, 2015; Lipp \& Purkis, 2006).

Lipp and Purkis (2006) proposed that extinction effects are found only when participants evaluate the CS prior to the CS-only presentations because the prior rating influences the strategy people use to evaluate the CS. If the first rating occurs only after the CS-only presentations (as done in between-participants designs), participants base their judgment on an integration of all the available evaluative information they encoded about the CS during the experiment. In contrast, when a prior rating occurred (as done in within-participants designs), participants base their judgment on the evaluative information that they received after their previous rating. Further, Gawronski et al. (2015) proposed that extinction effects in within-participants designs

\footnotetext{
${ }^{1}$ Previous articles have used the term "acquisition" for the procedure of displaying CS-US pairs, and "extinction" for the procedure of showing CS alone. However, because acquisition and extinction are also the common effects of these procedures, we chose to name those procedures after the stimulus presentation, and, when abbreviated, use "pairing" and "CS-only".
} 
do not reflect changes in the underlying evaluative representation of the CS, only a change in the evaluative verbal response of participants.

Gawronski et al. (2015) based their conclusion on the results of three studies. Because the present research is an extension of those studies, we describe them in detail. In Experiment 1, participants first observed pairings of two abstract shapes $\left(\mathrm{CS}_{\mathrm{pos}}\right)$ with positive images (e.g., puppies) and two abstract shapes ( $\left.\mathrm{CS}_{\text {neg }}\right)$ with negative images (e.g., a snake). After this pairing procedure, participants' evaluation of the CSs was measured using a self-reported evaluation question (how pleasant or unpleasant does the shape-image make you feel?). Then, participants observed a presentation of the CSs alone. After the CS-only presentations, the evaluation of the CSs was measured again with the same question. The results showed a significant extinction effect: EC effects (a preference for $\mathrm{CS}_{\text {pos }}$ over $\mathrm{CS}_{\text {neg }}$ ) were weaker post-CS-only than postpairing. Experiment 2 repeated the same procedure, with two changes: first, evaluation was measured indirectly, using the Evaluative Priming task (EPT; Fazio et al., 1995), and not only with self-report. Second, participants completed these measures only once: post-pairing or postCS-only (a between-participants design). The results showed no extinction effects on both measures (the EC effect was the same in both groups on both measures). In Experiment 3, all participants completed a self-report evaluation measure post-pairing and post-CS-only, and EPT once, post-CS-only. However, the first rating (post-pairing) was different between two groups: in one group, participants rated the CSs, whereas in the other group participants rated novel stimuli. All participants rated the CSs post-CS-only. Post-CS-only, the EC effect was weaker in the group that rated the CSs twice, post-pairing and post-CS-only, than in the group that rated the CSs for the first time post-CS-only (i.e., those who rated novel stimuli post-pairing). By contrast, 
evaluation measured with the EPT post-CS-only did not significantly differ between the two groups.

Overall, Gawronski et al. (2015) found extinction effects only when participants evaluated the CSs twice with the same question (once post-pairing and once post-CS-only). There was no evidence for an extinction effect when the CS evaluation was measured for the first time post-CS-only, or with an indirect evaluation measure - the EPT (the EC effect measured with the EPT was of the same size, regardless of whether the participants verbally rated the CS post-pairing or not). Gawronski et al. argued that the fact that only a direct evaluation measure (self-reported evaluation) but not an indirect evaluation measure (EPT) showed an extinction effect when participants rated the CS post-pairing suggests that the observed extinction effects on self-reported measures do not reflect real changes in the underlying evaluative representation of the CS, but rather reflect judgment-related processes that operate during the verbal expression of CS evaluations.

A different interpretation of Gawronski et al.'s (2015) results comes from a memorybased perspective on EC (Aust, Haaf, \& Stahl, 2019; Stahl \& Aust, 2018). This interpretation has much in common with the "changes in strategy" account suggested by Lipp and Purkis (2006) that we described above. According to the memory-based perspective, the same memory of the CS-US pairing can have different effects on different responses, depending on the strategy afforded by the measure. When participants are asked to evaluate the CS both post-pairing and post-CS-only, repeating the question creates a context that changes the strategy they use in their evaluation. Participants base their judgment on the evaluative information that they received after their previous rating (instead of basing their judgment on an integration of all the available evaluative information). According to this perspective, Gawronski et al.'s (2015) results suggest 
that the EPT is sensitive to the integration of all the available evaluative information, even when the context (the repeated direct measurement) changes the judgment in the direct evaluation questions (Aust et al., 2019).

One limitation of Gawronski et al.'s research is that they used only one indirect measure of evaluation, a measure that suffers from a very low sensitivity (i.e., high error variance; BarAnan \& Nosek, 2014; Gawronski \& De Houwer, 2014; Gawronski et al., 2015). Aust et al. (2019, p. 433) proposed that measuring direct evaluation before the CS-only trials might have failed to influence the EPT because of the measure's inferior reliability and sensitivity. In Gawronski et al.'s (2015) terms, perhaps the CS-only presentations led to a genuine change in the evaluative representation of the CS, but this change was detected only by the self-report measure and not by the EPT because the self-report is more sensitive to changes in evaluation than the EPT is. If this is correct, then an indirect measure with higher sensitivity would show an extinction effect. The goal of the present research was to test this possibility by using an indirect measure with higher sensitivity, the Implicit Association Test (IAT; Greenwald, McGhee, \& Schwartz, 1998).

\section{Preliminary Auxiliary Experiments}

For clarity, Table 1 summarizes the basic design of all the experiments in this research. Before testing the effect of post-pairing rating on post-CS-only EC effects, we first tested the robustness of the finding that, without post-pairing rating, there is no extinction in EC effects, using three different indirect measures: the EPT, the IAT, and the Affect Misattribution Procedure (AMP; Payne et al., 2005) and self-report evaluative ratings. In three experiments (Experiments S1-S3, one for each indirect measure), participants completed a self-reported evaluation measure and an indirect evaluation measure, either post-pairing or post-CS-only (i.e., 
a between-participants design). The full details of these experiments are reported in an online supplement (osf.io/4tg2y/). In the main text, we show the results in Figure 1. Replicating the results of Gawronski et al.'s (2015) Experiment 2, we did not find any evidence for extinction effects in any of the experiments, both in the direct and the indirect evaluation measures. These results extend previous evidence, using two measures (the AMP and the IAT) that have not been used before to test extinction effects in EC.

The online supplement reports another experiment (Experiment S4), in which we tested whether rating the CSs post-pairing leads participants to show extinction post-CS-only because repeating the exact same evaluation question is a communicative sign that the response should be different. To test that hypothesis, we manipulated the wording of the question (same wording or different wording), and examined whether changing the wording would eliminate the extinction effect. The full details of this experiment are reported in the online supplement (osf.io/4tg2y/). In the main text, we show the results in Figure 2. In contrast to the hypothesis, participants showed extinction effects regardless of the similarity in the wording of the questions. In the present context, Experiment S4 provided a replication of the finding that participants show extinction in the self-reported EC effect, when using a within-participants design, in which participants rate the CS post-pairing and post-CS-only.

\section{Overview of the Present Experiments}

Having confirmed (in Experiment S2) that the IAT does not show any evidence for an extinction effect when there is no post-pairing rating, we conducted two experiments to test whether the IAT would show an extinction effect when the CSs are rated post-pairing (i.e., in a within-participants design). In Experiment 1, we replicated Gawronski et al.'s (2015) Experiment 3, only replacing the EPT with the IAT (see Table 1). We compared post-CS-only EC effects as 
a function of whether, after the pairing and before the CS-only presentations, participants rated the CSs versus novel stimuli. Replicating Gawronski et al.'s finding with the EPT, we found that evaluation measured with the IAT post-CS-only did not significantly differ as a function of whether participants rated the CSs versus novel stimuli post-pairing. However, unlike Gawronski et al.'s results, the self-reported EC also did not significantly differ between the two conditions. Interestingly, the EC effect measured with self-reported rating was weaker post-CS-only than post-pairing, in both groups: among participants who rated the CSs post-pairing and among participants who rated novel stimuli post-pairing. That is, both conditions showed an extinction effect.

Experiment 1's results suggested that any evaluative rating of stimuli post-pairing-be those stimuli the CSs or novel stimuli-leads to extinction effects on direct evaluation measures. To test that possibility, in Experiment 2, we added another group that did not rate any stimuli post-pairing (see Table 1). We compared the post-CS-only EC effect in this group to the postCS-only EC effect among participants who rated novel stimuli post-pairing and among participants who rated the CS post-pairing. We tested whether indeed an extinction effect is observed whenever participants rate any stimuli post-pairing, but not when they do not rate any stimuli post-pairing.

Another purpose of Experiment 2 was to understand better the IAT results in Experiment 1. In Experiment 1, evaluation measured with the IAT post-CS-only did not significantly differ between the two conditions (rating of the CSs versus rating of novel stimuli post-pairing). Originally, we thought that such results would be compatible with the conclusion that the extinction effects after rating the CSs pre-extinction, can be found only with self-report (direct) evaluation measures. However, because in Experiment 1, self-reported EC showed an extinction 
effect in both conditions (after rating novel stimuli and after rating the CSs, post-pairing), the lack of a difference between the two conditions in the EC effect measured with the IAT was uninformative. Perhaps the IAT showed an extinction effect in both conditions, just like the selfreport measure, or maybe it did not show extinction in any of the conditions. Without a postpairing IAT measurement, we were unable to distinguish between these two possibilities. In Experiment 2, we added another group of participants who completed the IAT only post-pairing. We then compared the IAT effect in that condition, to the IAT effect in the other three conditions, to learn whether the IAT showed an extinction effect in any of the conditions.

We report all data exclusions, manipulations, measures, and how we determined our sample sizes. In all experiments, decisions to stop collecting data did not depend on the obtained results. We pre-registered the materials, target sample size, exclusion rules, and analysis plans (links to the preregistration are presented below for each study). To see the materials and data of the whole project visit osf.io/xuhpt/. 
Table 1

Procedure of Experiments: Post-pairing and post-CS-only measurement.

\begin{tabular}{|c|c|c|c|}
\hline Experiment & Group & Post-pairing measurement & Post-CS-only measurement \\
\hline \multirow{2}{*}{$\mathrm{S} 1$} & $\begin{array}{l}\text { Post-pairing } \\
\text { measurement }\end{array}$ & $\begin{array}{l}\text { CS rating } \\
\text { EPT }\end{array}$ & - \\
\hline & $\begin{array}{l}\text { Post-CS-only } \\
\text { measurement }\end{array}$ & - & $\begin{array}{l}\text { CS rating } \\
\text { EPT }\end{array}$ \\
\hline \multirow{2}{*}{ S2 } & $\begin{array}{l}\text { Post-pairing } \\
\text { measurement }\end{array}$ & $\begin{array}{l}\text { CS rating } \\
\text { IAT }\end{array}$ & - \\
\hline & $\begin{array}{l}\text { Post-CS-only } \\
\text { measurement }\end{array}$ & - & $\begin{array}{l}\text { CS rating } \\
\text { IAT }\end{array}$ \\
\hline \multirow{2}{*}{ S3 } & $\begin{array}{l}\text { Post-pairing } \\
\text { measurement }\end{array}$ & $\begin{array}{l}\text { CS rating } \\
\text { AMP }\end{array}$ & - \\
\hline & $\begin{array}{l}\text { Post-CS-only } \\
\text { measurement }\end{array}$ & - & $\begin{array}{l}\text { CS rating } \\
\text { AMP }\end{array}$ \\
\hline \multirow{2}{*}{ S4 } & $\begin{array}{l}\text { Same rating } \\
\text { question }\end{array}$ & $\begin{array}{l}\text { CS rating (liking or } \\
\text { pleasantness) }\end{array}$ & $\begin{array}{c}\text { CS rating (same question) } \\
\text { IAT }\end{array}$ \\
\hline & $\begin{array}{c}\text { Different rating } \\
\text { questions }\end{array}$ & $\begin{array}{l}\text { CS rating (liking or } \\
\text { pleasantness) }\end{array}$ & $\begin{array}{l}\text { CS rating (different question) } \\
\text { IAT }\end{array}$ \\
\hline \multirow{2}{*}{1} & CS rating & CS rating & $\begin{array}{l}\text { CS rating } \\
\text { IAT }\end{array}$ \\
\hline & Novel rating & Novel stimuli rating & $\begin{array}{l}\text { CS rating } \\
\text { IAT }\end{array}$ \\
\hline \multirow{4}{*}{2} & $\mathrm{CS}$ rating & CS rating & $\begin{array}{l}\text { CS rating } \\
\text { IAT }\end{array}$ \\
\hline & Novel rating & Novel stimuli rating & $\begin{array}{l}\text { CS rating } \\
\text { IAT }\end{array}$ \\
\hline & No rating & - & $\begin{array}{l}\text { CS rating } \\
\text { IAT }\end{array}$ \\
\hline & IAT & IAT & - \\
\hline
\end{tabular}



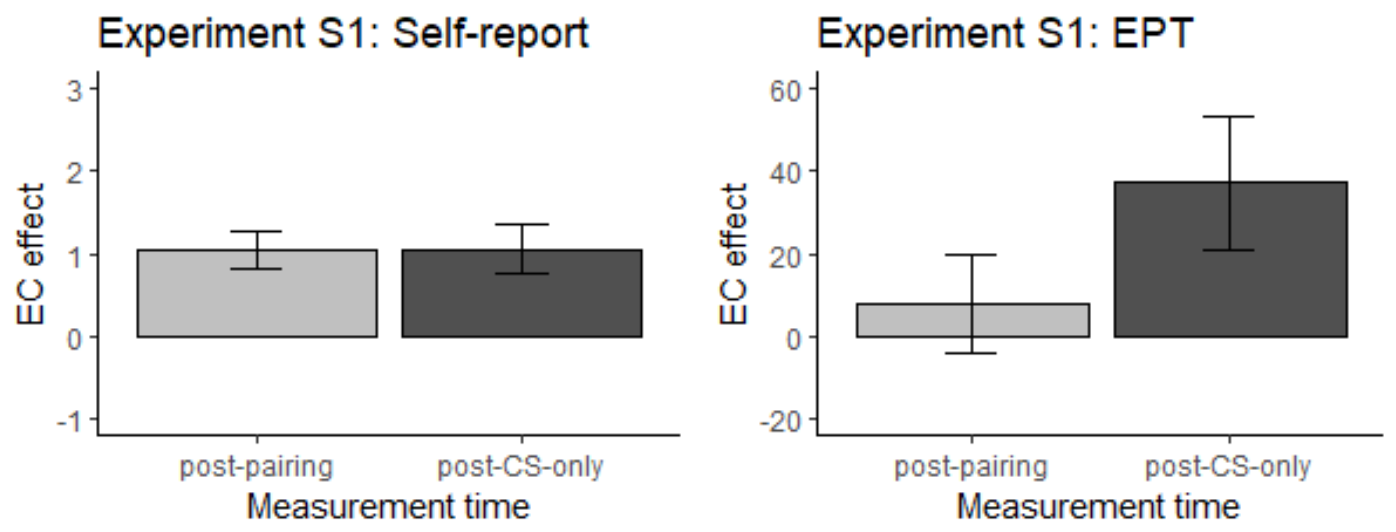

Experiment S2: Self-report
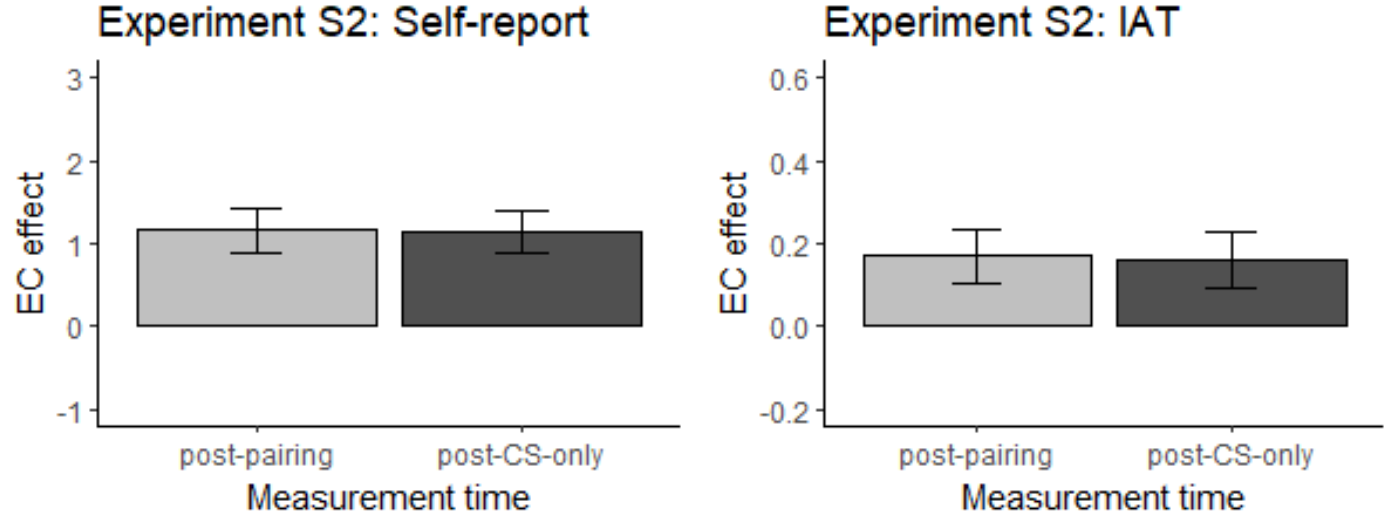

Experiment S3: Self-report
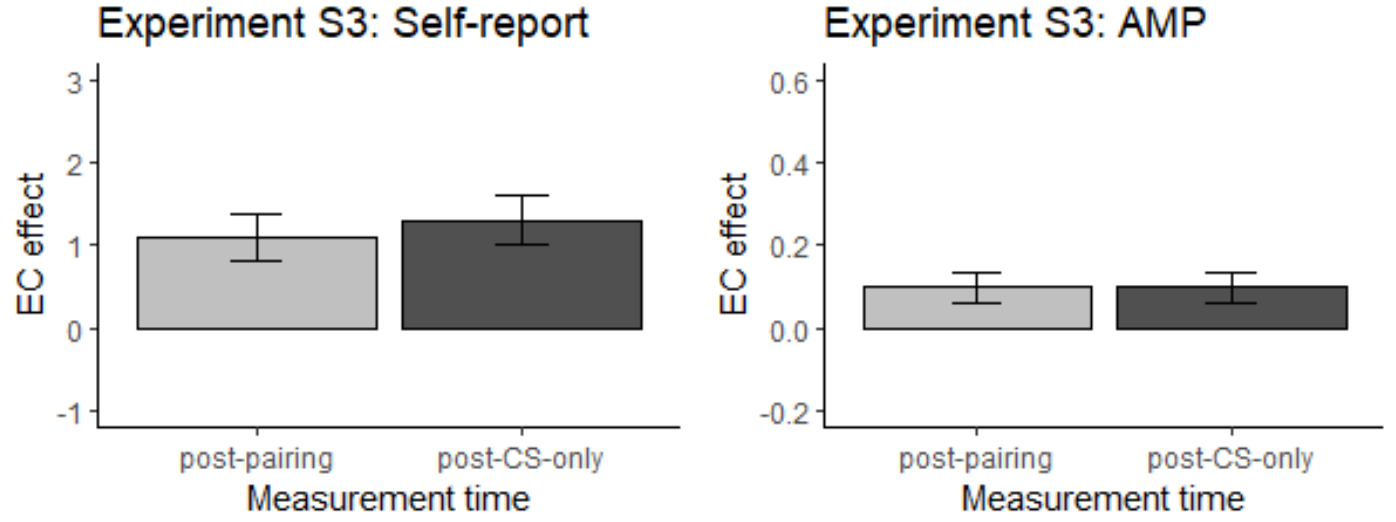

Figure 1. Experiments S1-S3: EC effects (preference for $\mathrm{CS}_{\mathrm{pos}}$ over $\mathrm{CS}_{\text {neg }}$ ) as a function of measurement time (post-pairing, post-CS-only) and measure (self-report, indirect). The upper Graph presents the result for Experiment S1 (with the EPT as indirect measure). The middle Graph presents the result for Experiment S2 (with the IAT as indirect measure). The lower Graph presents the result for Experiment S3 (with the AMP as indirect measure). Error-bars represent $95 \%$ confidence intervals. 


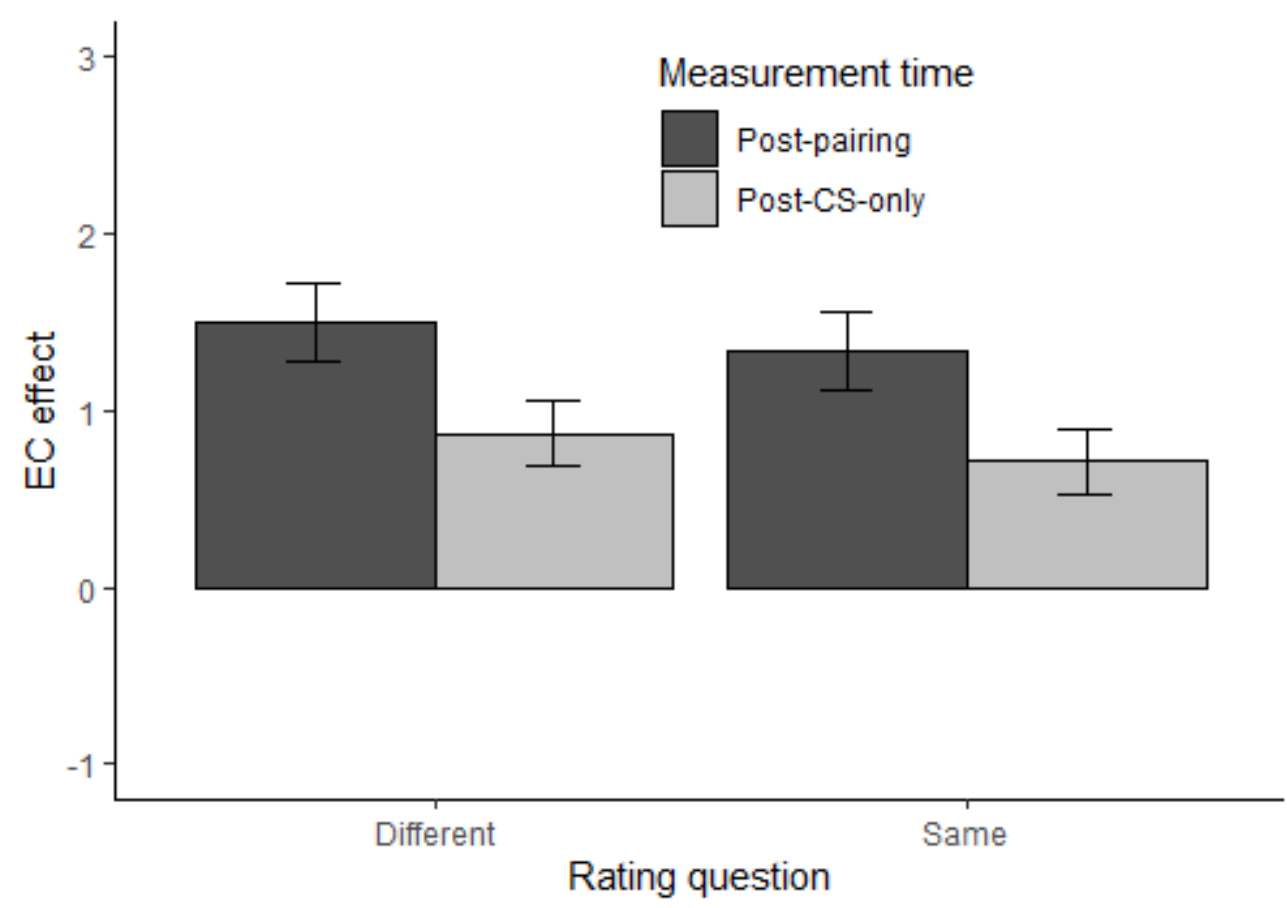

Figure 2. Experiment S4: EC effects (preference for $\mathrm{CS}_{\mathrm{pos}}$ over $\mathrm{CS}_{\text {neg }}$ ) as a function of measurement time (post-pairing, post-CS-only) and rating question (different, same). Error-bars represent $95 \%$ confidence intervals.

\section{Experiment 1}

\section{Method}

Participants. The pre-registration for Experiment 1 is available at osf.io/bxcsg.

Participants in all experiments volunteered to participate online at the Project Implicit research website (Nosek, 2005). Based on power-analysis we estimated that 1,200 participants would allow power of $95 \%$ to detect an effect of $d=0.2$ in the IAT t-test (the comparison between the 
two post-pairing ratings conditions). ${ }^{2} 1,212$ participants completed the experiment (out of the 1,892 who started it, $64 \%$; this is a typical completion rate at Project Implicit, perhaps because the participation is completely voluntary). Four participants were excluded because they did not answer all the evaluation questions. An additional 18 participants who had more than $10 \%$ fast trials in the IAT were excluded. ${ }^{3}$ The final sample included 1,190 participants $(65 \%$ women, $\left.M_{\text {age }}=34.03, S D=15.10\right)$.

Materials and procedure. Participants first completed a CS-US pairings procedure. Next, participants completed a self-report measure of evaluation. Half of the participants rated the pleasantness of the CSs and the remaining rated five novel stimuli. After that, participants observed the CS-only presentations. Finally, in a random order, participants evaluated the CSs on a self-report measure and completed an IAT. ${ }^{4}$

CS-US pairings and CS-only presentations. We adopted the procedures and materials used in Gawronski et al. (2015). CSs were computer-generated images of shapes. USs were 16 positive and 16 negative images from the International Affective Picture System (Lang, Bradley, \& Cuthbert, 2008) and photos found in a web search. Two CSs co-occurred with positive USs, and two CSs co-occurred with negative USs. A fifth CS appeared alone on the screen and served as a baseline CS. Each trial began with a fixation cross presented for $250 \mathrm{~ms}$, followed by a CS

${ }^{2}$ To save resources, we planned to stop at 300,600, and 900 participants and test the Bayes factor for both critical comparisons. If $\mathrm{BF}_{01}$ or $\mathrm{BF}_{10}$ in both t-tests is above 10 , we planned to stop collecting the data.

${ }^{3}$ Although these exclusions were not preregistered, we included them in all experiments, because they are the common practice when using data from Project Implicit and when using the IAT in general (e.g., BarAnan \& Nosek, 2014; Greenwald et al., 2003). Including the excluded participants does not change the results.

${ }^{4}$ Measure order did not moderate any of the effects. 
and a US appearing together on the screen for 1500ms. On half of the trials, the CS was on the left (and the US was on the right), and on the other half the CS was on the right side of the screen. The inter-trial-interval was $1500 \mathrm{~ms}$. The assignment of the CSs to paired valence was selected randomly for each participant. Each CS was paired once with each of eight USs (different photos of the same valence), and the baseline CS appeared alone eight times, resulting in a total of 40 trials. The CS-only presentations procedure was similar to the pairings procedure, the only difference being that the five CSs were presented alone (without the USs).

Self-reported evaluation. After the CS-US pairing procedure, we asked one group of participants to rate each CS by answering the question "Please rate how pleasant or unpleasant each image makes you feel" with response options that ranged from $1=$ very unpleasant to $7=$ very pleasant . We asked the other group of participants to rate, using the same measure, five unfamiliar stimuli (from the same set of computer-generated drawings, adopted from Gawronski et al., 2015). After the CS-only presentations procedure, all participants rated the pleasantness of each of CSs using the same measure that we used post-pairing.

IAT. The two category stimuli in the IAT were one $\mathrm{CS}_{\text {pos }}$ and one $\mathrm{CS}_{\text {neg }}$ (each randomly selected from the two CSs of that role). The attribute stimuli were eight positive words (randomly selected for each participant from Pleasant, Good, Outstanding, Beautiful, Magnificent, Marvelous, Excellent, Appealing, Delightful, Nice) and eight negative words (randomly selected from Unpleasant, Bad, Horrible, Miserable, Hideous, Dreadful, Painful, Repulsive, Awful, Ugly). In the IAT, participants categorize the stimuli using two computer keys. The category labels appear on the upper left and upper right corners of the screen (For the CSs, the category labels were photos of the images). In the critical blocks, participants responded with one key to the stimuli of two categories (e.g., the picture of the $\mathrm{CS}_{\text {pos }}$ and "Good words") and 
responded with the other key to the stimuli that belonged to the other two categories (e.g., picture of the $\mathrm{CS}_{\text {neg }}$ and "Bad words"). The IAT consisted of seven blocks and followed the procedure described in Nosek, Greenwald, and Banaji (2005). With the R's IAT package (Martin, 2016), we computed the IAT D score (Greenwald et al., 2003) such that a score above zero reflected a preference for $\mathrm{CS}_{\text {pos }}$ over the $\mathrm{CS}_{\text {neg. }}$. Based on the IAT scores from three parcels of the IAT critical blocks, internal consistency (Cronbach's $\alpha$ ) was .83 .

Design. The design was 2 (CS valence: positive, negative; within participants) x 2 (Postpairing ratings: CSs, novel stimuli; between participants) x 2 (Evaluation measure: self-report, IAT; within participants) x 2 (Measurement time: post-pairing, post-CS-only; within participants)..$^{5}$

\section{Results}

Self-report. Following Gawronski et al. (2015), participants' ratings of the baseline CS were subtracted from their ratings of each of the four CSs that had been paired with a positive or negative US. The resulting difference scores were then aggregated by averaging the scores of the two CSs that had been paired with a US of the same valence (Cronbach's $\alpha$ s $=.71, .71$ for postpairing $\mathrm{CS}_{\mathrm{pos}}$ and $\mathrm{CS}_{\text {neg }}$, respectively, and $.66, .65$ for post-CS-only $\mathrm{CS}_{\text {pos }}$ and $\mathrm{CS}_{\text {neg, }}$, respectively). We computed a preference (EC) score for $\mathrm{CS}_{\text {pos }}$ over $\mathrm{CS}_{\text {neg }}$ for each period of the experiment (post-pairing and post-CS-only) by subtracting the mean rating for $\mathrm{CS}_{\text {neg }}$ from the mean rating for $\mathrm{CS}_{\mathrm{pos}}$ in that period. Table 2 presents the self-reported evaluation scores as a function of CS valence and the kind of CSs participants rated post-pairing. We did not replicate Gawronski et al.'s findings. The post-CS-only EC score when participants had rated the CSs

\footnotetext{
${ }^{5}$ Measurement time factor is relevant only for the group that rated the CSs post-pairing.
} 
post-pairing $(M=0.69, S D=1.61)$ was not significantly different from the post-CS-only EC score when participants had rated novel stimuli post-pairing $(M=0.76, S D=1.78), t(1,184.6)=$ $0.753, p=.451, d=0.04$. Because many of the comparisons of interest reported in this manuscript were not significant, we also report a Bayesian analysis for each comparison in Table 3. To allow a more accurate interpretation of the Bayesian analysis, Table 3 details the Bayesfactors for each comparison, assuming different effect sizes (i.e., different priors).

Next, we explored the possibility that both conditions showed a reduction in the EC effect. To test this, we compared the post-CS-only preference (EC) scores in each of the conditions to the post-pairing preference (EC) score. For the group that rated the CSs postpairing we tested that comparison with a paired-samples t-test ${ }^{6}$, and found a weaker EC score post-CS-only $(M=0.69, S D=1.61)$ than post-pairing $(M=1.21, S D=1.88), t(579)=7.90, p$ $<.001, d=0.32$. For the group that rated novel stimuli post-pairing, we tested that comparison with an independent-samples t-test, and found a weaker EC score post-CS-only $(M=0.76, S D=$ 1.78) than post-pairing $(M=1.21, S D=1.88), t(1,173.6)=4.19, p<.001, d=0.24$. Thus, we found a decrease in EC effects in both conditions.

IAT. Table 2 presents the IAT scores as a function of the stimuli that were rated after the pairing. The IAT scores in both groups were significantly different from zero (indicating an EC effect). The IAT score when participants had rated the CSs post-pairing $(M=0.11, S D=0.44)$ was not significantly different from the IAT score when participants had rated novel stimuli postpairing $(M=0.10, S D=0.45), t(1187.5)=-0.27, p=.396, d=-0.02$.

\footnotetext{
${ }^{6}$ We excluded from this analysis one participant who did not rate all CSs post-pairing.
} 


\section{Discussion}

In Experiment 1, replicating what Gawronski et al. (2015) found using an EPT, we found that evaluation measured with the IAT post-CS-only did not significantly differ as a function of whether participants rated the CSs versus novel stimuli post-pairing. Bayesian analysis showed moderate evidence for a null effect even when assuming a small effect size (Table 3). However, it is difficult to interpret this result because, unlike Gawronski et al., the self-reported EC effect in our study was not different between the two conditions. Interestingly, the post-CS-only EC effect was weaker than the post-pairing EC effect, both when participants had rated the CSs (i.e., in a within-participants comparison) and when participants had rated novel stimuli post-pairing (i.e. in a between-participants comparison), suggesting that both conditions led to an extinction effect. This raises the possibility that an extinction effect of EC on self-reported measures occurs whenever participants make an evaluative rating before the CS-only presentations, no matter what they evaluate. Experiment 2 tested that possibility by comparing the extinction effect on a self-report measure when participants rated novel targets post-pairing, to the effect of extinction when participants did not rate any stimuli until they rated the CSs at post-CS-only. Moreover, we tested the possibility that evaluation measured with the IAT, like the self-reported evaluation, shows an extinction effect (i.e., reduced EC effect) when participants make (any) evaluative rating post-pairing. In Experiment 2, we compared EC effects on the IAT at post-CS-only after participants evaluated the CS post-pairing, to EC effects on the IAT measured post-pairing. 
Table 2

Experiment 1: Self-reported evaluations and IAT scores as a function of CS valence, measurement time, and post-pairing ratings.

\begin{tabular}{|c|c|c|c|c|c|}
\hline \multirow{2}{*}{$\begin{array}{c}\text { Measurement } \\
\text { time }\end{array}$} & \multirow[t]{2}{*}{$\mathrm{N}$} & \multicolumn{3}{|c|}{ Self-report } & \multirow{2}{*}{$\begin{array}{c}\text { IAT } \\
\text { IAT Score }\end{array}$} \\
\hline & & $\begin{array}{c}\text { Positive } \\
\text { CS }\end{array}$ & $\begin{array}{c}\text { Negative } \\
\text { CS }\end{array}$ & EC Score & \\
\hline \multicolumn{6}{|c|}{ Group 1: Rated the CS post-pairing } \\
\hline Post-pairing & 580 & $\begin{array}{c}4.60 \\
(1.20)\end{array}$ & $\begin{array}{c}3.39 \\
(1.17)\end{array}$ & $\begin{array}{c}1.21(1.88) \\
t(579)=15.42 \\
p<.001, d=0.64\end{array}$ & \\
\hline Post-CS-only & 581 & $\begin{array}{c}4.30 \\
(1.08)\end{array}$ & $\begin{array}{c}3.61 \\
(1.08)\end{array}$ & $\begin{array}{c}0.69(1.61) \\
t(580)=10.34 \\
p<.001, d=0.42\end{array}$ & $\begin{array}{c}0.11(0.44) \\
t(580)=6.40, \\
p<.001, d=0.26\end{array}$ \\
\hline \multicolumn{6}{|c|}{ Group 2: Rated novel stimuli post-pairing } \\
\hline Post-CS-only & 609 & $\begin{array}{c}4.42 \\
(1.12)\end{array}$ & $\begin{array}{c}3.65 \\
(1.13)\end{array}$ & $\begin{array}{c}0.76(1.78) \\
t(608)=10.59 \\
p<.001, d=0.43\end{array}$ & $\begin{array}{c}0.10(0.45) \\
t(608)=5.82 \\
p<.001, d=0.23\end{array}$ \\
\hline
\end{tabular}


Table 3

Experiments 1-2: Bayesian analysis $\left(B F_{10}\right)$ for the reported comparisons, as a function of priors (assumed effect sizes)

\begin{tabular}{|c|c|c|c|c|}
\hline $\begin{array}{c}\text { Experiment } \\
\text { (measure) }\end{array}$ & Comparison & $d=0.3$ & $\mathrm{~d}=0.5$ & $\mathrm{~d}=0.707 *$ \\
\hline \multirow[t]{3}{*}{1 (Self-report) } & $\begin{array}{l}\text { Post-CS-only preference: CS rating } \\
\text { vs. Novel rating }\end{array}$ & 0.194 & 0.120 & 0.085 \\
\hline & $\begin{array}{l}\text { CS rating: post-CS-only preference } \\
\text { vs. post-pairing preference }\end{array}$ & $4.12 \mathrm{e}+11$ & $3.76 e+11$ & $3.13 e+11$ \\
\hline & $\begin{array}{l}\text { Novel rating: post-CS-only } \\
\text { preference vs. post-pairing } \\
\text { preference }\end{array}$ & 593.01 & 470.39 & 367.7 \\
\hline 1 (IAT) & $\begin{array}{l}\text { Post-CS-only preference: CS rating } \\
\text { vs. Novel rating }\end{array}$ & 0.160 & 0.098 & 0.070 \\
\hline \multirow[t]{3}{*}{2 (Self-report) } & $\begin{array}{l}\text { Post-CS-only preference: No rating } \\
\text { vs. Novel rating } \\
\end{array}$ & 28.74 & 24.36 & 19.73 \\
\hline & $\begin{array}{l}\text { Post-CS-only preference: No rating } \\
\text { vs. CS rating }\end{array}$ & 46.81 & 40.90 & 33.60 \\
\hline & $\begin{array}{l}\text { Post-CS-only preference: CS rating } \\
\text { vs. Novel rating }\end{array}$ & 0.231 & 0.146 & 0.105 \\
\hline \multirow[t]{4}{*}{2 (IAT) } & $\begin{array}{l}\text { IAT post-pairing vs. IAT post-CS- } \\
\text { only: CS rating }\end{array}$ & 0.461 & 0.306 & 0.224 \\
\hline & $\begin{array}{l}\text { IAT post-pairing vs. IAT post-CS- } \\
\text { only: Novel rating }\end{array}$ & 0.262 & 0.167 & 0.120 \\
\hline & $\begin{array}{l}\text { IAT post-CS-only: CS rating vs. No } \\
\text { rating }\end{array}$ & 7.72 & 6.19 & 4.89 \\
\hline & $\begin{array}{c}\text { IAT post-CS-only: Novel rating vs. } \\
\text { No rating }\end{array}$ & 0.340 & 0.221 & 0.160 \\
\hline
\end{tabular}

Note. $B F_{10}$ is the support for $\mathrm{H} 1$ over $\mathrm{H} 0$ (the likelihood of the data under the alternative

hypothesis compared to the null hypothesis). $* d=0.707$ is the default prior for the for the ttestBF function ('BayesFactor' package; Morey et al., 2018).

\section{Experiment 2}

\section{Method}

Participants. The pre-registration for Experiment 2 is available at osf.io/mjkb6. Based on power-analysis we estimated that 1,000 participants (250 participants in each of the critical between-participants conditions) will allow power of more than $95 \%$ to detect an effect of $d=$ 
0.3 for a comparison between two independent groups (the main comparison was between the IAT and CS rating conditions, see Table 1). ${ }^{7} 1,007$ participants completed the experiment (out of 1,480 who started it, 68\%). We excluded six participants because they did not answer all the rating questions, and six participants because they had more than $10 \%$ fast trials $(\mathrm{RT}<300$; Greenwald et al., 2003) in the IAT. The final sample included 995 participants (61\% women, $\left.M_{\text {age }}=38.17, S D=14.12\right)$.

Materials, procedure, and design. Participants first completed a CS-US pairing procedure similar to the one in Experiment 1. Next, participants were randomly assigned to one of four conditions. Two groups were the same as in Experiment 1: One group rated the CSs, and another group rated novel stimuli. A third group completed an IAT, and the fourth group of the participants continued immediately to the CS-only presentations phase, without completing any measure post-pairing. Those who completed the IAT, ended the experiment at this stage. All the other participants observed the CS-only presentations, as in Experiment 1. These participants ended the study with the two, randomly-ordered measures of CS evaluation: self-report and an IAT. The post-CS-only self-report measure and the IAT were similar to those used in Experiment 1.

\section{Results}

Self-report. We computed the evaluation scores as in Experiment 1. The internal consistency of the post-CS-only self-report evaluation was $\alpha$ Cronbach $=.69, .67$ for post-pairing

\footnotetext{
${ }^{7}$ To save resources, we planned to stop at 500 and add 500 if the results are not significant for the two confirmatory tests. Therefore, we used $p=.027$, instead of $p=.05$, as our critical p-value (Sagarin, Ambler, \& Lee, 2014). We changed the sequential testing strategy because we were not sure that the strategy used in Experiment 1 is recommended when the main hypothesis testing follows NHST.
} 
$\mathrm{CS}_{\mathrm{pos}}$ and $\mathrm{CS}_{\mathrm{neg}}$, respectively, and $.72, .70$ for post-CS-only $\mathrm{CS}_{\mathrm{pos}}$ and $\mathrm{CS}_{\text {neg }}$, respectively. Table 4 presents the self-reported evaluation scores as a function of CS valence and post-pairing ratings condition. We planned direct comparisons between specific groups for testing our research questions. The results suggested extinction in both conditions in which participants rated stimuli post-pairing: The post-CS-only EC score when participants did not rate any stimuli post-pairing $(M=1.33, S D=2.09)$ was larger than when participants had rated novel stimuli post-pairing $(M$ $=0.77, S D=1.63), t(463.13)=3.13, p=.001, d=0.23$, and larger than when participants had rated the CSs post-pairing $(M=0.74, S D=1.55), t(451.11)=3.51, p<.001, d=0.32$.

Replicating the results of Experiment 1, we found no significant difference in the size of postCS-only EC score (i.e., in the size of the extinction effect) between participants who rated the CSs and participants who rated novel stimuli post-pairing, $t(470.22)=0.23, p=.818, d=0.02 .^{8}$ In summary, the evidence suggests that people report weaker EC effect post-CS-only, if they rated any stimuli post-pairing than if they rated no stimuli at all.

IAT. Based on the IAT scores from three parcels of the IAT critical blocks, internal consistency of the IAT was $\alpha=.86$. Table 4 presents the IAT scores as a function of post-pairing rating. The IAT scores in all the conditions were significantly different from zero (indicating an EC effect). We planned direct comparisons between specific groups for testing our research questions. The main planned test did not find evidence for an extinction effect: The IAT score of

\footnotetext{
${ }^{8}$ Exploratory comparisons raveled that the post-pairing EC score $(M=1.37, S D=1.84)$ of the participants who rated the CSs post-pairing was stronger than the post-CS-only EC score of those participants $(M=0.74, S D=1.55), t(225)=6.19, p<.001, d=0.41, B F_{10}=2949833$, and, stronger than the post-CS-only EC score of the participants who rated novel stimuli post-pairing $(M=0.77, S D=1.63)$, $t(451.15)=3.71, p<.001, d=0.34, B F_{10}=83.57$, but not stronger than the post-CS-only EC score of the participants who did not evaluate any stimuli post-pairing $(M=1.33, S D=2.09), t(469.28)=0.214, p=$ $0.830, d=0.01, B F_{10}=0.104$. Bayes-factors in this section were computed with the default prior.
} 
participants who completed the IAT post-pairing $(M=0.13, S D=0.46)$ was not larger than the IAT score of participants who completed the IAT post-CS-only, after rating the CSs post-pairing $(M=0.08, S D=0.48), t(471.21)=1.28, p=.198, d=0.11$, and not different from the IAT score of participants who completed the IAT post-CS-only, after rating novel stimuli post-pairing $(M=$ $0.16, S D=0.50), t(502.51)=-0.65, p=.511, d=-0.05$. A secondary test for an extinction effect did find suggestive evidence of an extinction of the indirectly measured EC: The IAT score of participants who completed the IAT post-CS-only after rating the CSs post-pairing $(M=0.08$, $S D=0.48)$ was smaller than the IAT score of participants who completed the IAT post-CS-only but did not rate any stimuli post-pairing $(M=0.21, S D=0.48), t(466.78)=2.83, p=.004, d=$ 0.26. The IAT score of participants who completed the IAT post-CS-only, after rating novel stimuli post-pairing was not smaller than the IAT score of participants who completed the IAT post-CS-only, after not rating any stimuli post-pairing, $t(490.61)=0.98, p=.323, d=0.08$. The subscripts in Table 4 succinctly show the comparisons of the IAT scores between the four groups. ${ }^{9}$

\section{Discussion}

In Experiment 2, we examined two questions. We examined whether, as suggested by the surprising result of Experiment 1, CS-only presentations decreases self-reported EC effects, as long as participants rate any stimuli prior to the CS-only presentations (post-pairing), no matter what stimuli they evaluate. Supporting that possibility, we found a smaller EC score (reflecting a larger extinction effect) when participants rated novel stimuli post-pairing and when participants

\footnotetext{
${ }^{9}$ Measure order did not influence the results of these comparisons.
} 
rated the CSs post-pairing, in comparison to the EC score of participants who did not evaluate any stimuli post-pairing.

Our second question was whether an indirect evaluation measure that does not suffer from low reliability would show evidence for a reduced EC effect, if participants evaluated stimuli before the CS-only presentations (post-pairing). We did not find evidence that the indirectly measured EC effect was larger before the CS-only presentations than after this procedure, even when participants rated stimuli before the CS-only presentations. Bayesian analysis showed moderate evidence for a null effect when assuming a medium effect size but only anecdotal evidence for a null effect when assuming a small effect size (see Table 3 ). We did find a smaller post-CS-only IAT score (i.e., a larger extinction effect) among participants who rated the CSs post-pairing than among participants who did not rate any stimuli post-pairing. That finding might serve as evidence for an extinction effect on the IAT. However, that evidence is rather weak because the effect was small and because the critical evidence for an extinction effect was not found: the IAT score of participants who complete the IAT post-CS-only and rated the CSs post-pairing was not significantly different than the IAT score of the participants who completed the IAT only post-pairing, without undergoing any extinction procedure. 
Table 4

Experiment 2: Self-reported evaluations and IAT scores as a function of CS valence, measurement time, and post-pairing rating condition.

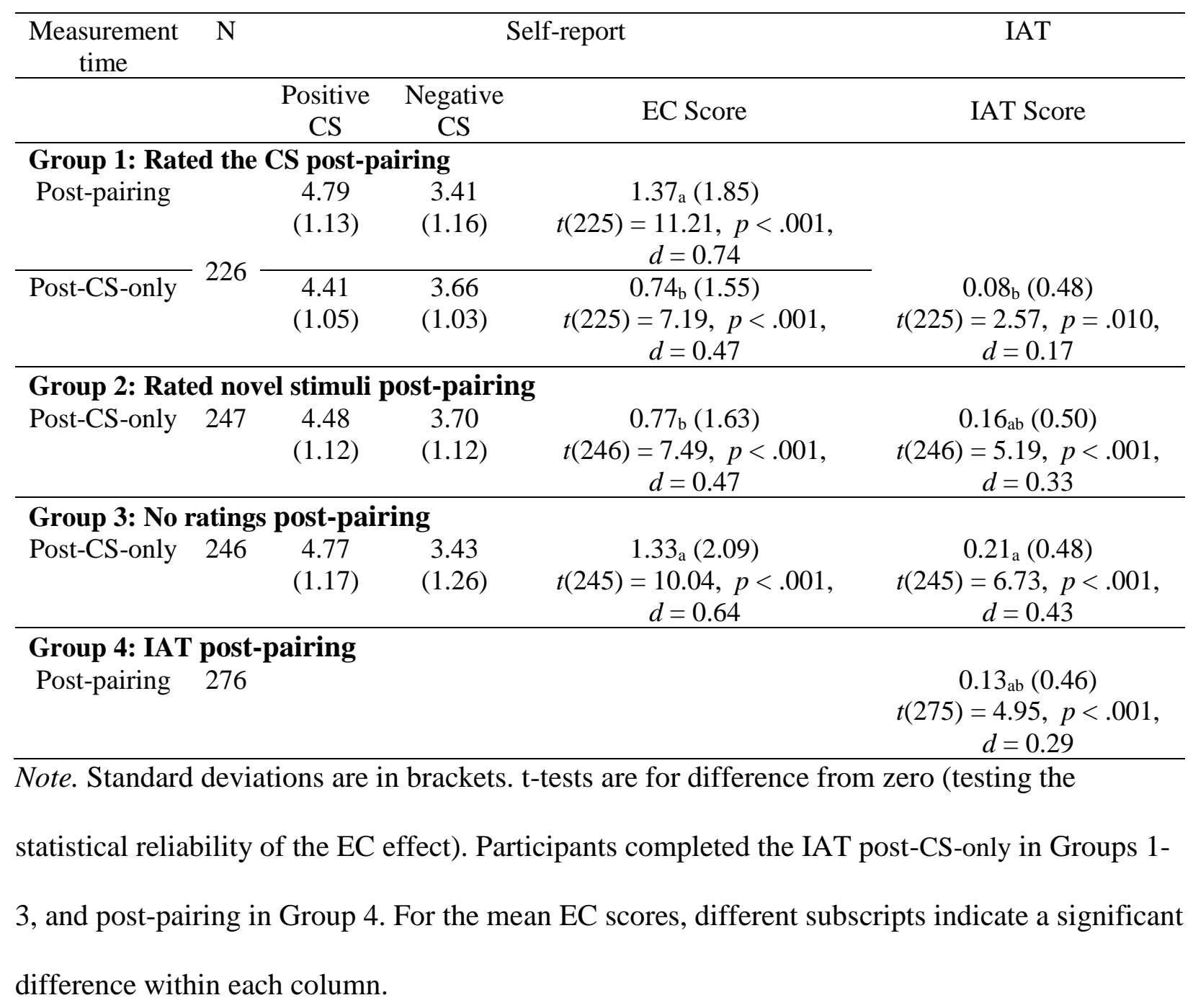




\section{General Discussion}

In the present research, we replicated and extended studies from previous research (Gawronski et al., 2015) that found an extinction effect of EC after CS-only presentations only when participants rated the CS before the CS-only presentations, but not when CS evaluation was measured only post-CS-only or indirectly with EPT (Fazio et al., 1995). These results suggest that evaluation measured indirectly is not sensitive to extinction. However, inference from a lack of an effect is weak, especially when the measure suffers from low reliability. Therefore, in the present research, we searched for evidence of extinction with a more reliable indirect measure, the IAT (Greenwald et al., 1998).

Experiment 1 was a straightforward replication of the previous research (Experiment 3 in Gawronski et al, 2015). Participants completed the IAT post-CS-only, after either rating the CSs post-pairing (which was supposed to cause extinction in self-reported evaluation) or rating novel stimuli post-pairing (which was not supposed to cause extinction in self-reported evaluation). Replicating with the IAT what Gawronski et al. found with EPT, we found that evaluation measured with the IAT post-CS-only did not significantly differ as a function of whether participants rated the CSs or novel stimuli post-pairing. However, unlike the results of Gawronski et al., in our experiment, the self-reported EC effect also did not significantly differ between the two conditions. Moreover, we found that self-reported EC effect was weaker postCS-only than post-pairing both when participants had rated the CSs and when participants had rated novel stimuli post-pairing, suggesting that both conditions led to an extinction effect.

Because Experiment 1 found the same results in self-reported EC regardless of the stimuli rated post-pairing, together with suggestive evidence that both conditions showed extinction, there are two possible interpretations for the lack of difference between the two 
conditions in the IAT. It could be that the IAT showed no extinction effect in both conditions, just as the EPT in the original study. Or, perhaps, just like the self-report measure in our experiment, the IAT showed an extinction effect in both conditions.

Experiment 2 pursued stronger evidence that, indeed, there is an extinction effect in selfreported EC, whenever participants rate any stimuli post-pairing, by adding a control group that did not rate any stimuli post-pairing (rated the CS only post-CS-only). Experiment 2 also pursued a stronger test for an extinction effect (or the lack thereof) in the IAT, by adding another control group that completed the IAT post-pairing. In Experiment 2, we found a larger extinction effect on self-reported EC when participants rated novel stimuli post-pairing than when they did not rate any stimuli post-pairing. That result suggested that, compatible with the suggestive evidence from Experiment 1, rating any stimuli post-pairing leads to a decrease in EC effect on self-reported ratings post-CS-only. As for the IAT, in Experiment 2, we found that the IAT score measured at post-pairing was not significantly different from the IAT score measured post-CSonly, regardless of whether, post-pairing, participants rated any stimuli or not.

We did find a smaller post-CS-only IAT effect when participants rated the CSs postpairing than when participants did not rate any stimuli post-pairing. However, this effect was small, and the critical comparison for an extinction effect (post-pairing vs. post-CS-only, between participants) revealed no evidence for extinction. Therefore, we do not think we found good evidence for an extinction effect on the IAT.

\section{Theoretical Implications}

The results of the present research are compatible with the conclusion that extinction effects of EC are limited to cases in which evaluation is self-reported, and another self-report rating (of the same stimuli or other stimuli) occurred after the pairing procedure and before the 
CS-only presentations. In line with Gawronski et al.'s results, the present results do not provide any evidence for extinction effects (regardless of the context) when evaluation is measured with indirect measures. Our research increases the confidence that extinction of EC does not occur for indirectly measured evaluation because we used a different indirect measure (the IAT) that often shows better sensitivity than the EPT used in Gawronski et al.'s research.

The meaning of the present results depends on one's perspective about the previous findings. Our IAT results are compatible with Gawronski et al.'s (2015) conclusions that extinction effects on self-report do not reflect real changes in evaluative representation that occur before the self-report measurement. Rather, they reflect judgment-related processes that operate during the verbal expression of CS evaluations. Alternatively, according to the memory-based perspective (e.g., Aust et al., 2019), the current IAT results suggest that the adaptation of judgment strategies due to the context of post-pairing rating is limited to direct, self-reported evaluation measures.

The exact reason why extinction of EC occurs for self-report measures is still unknown. In contrast to Gawronski et al. (2015), who found stronger post-CS-only self-reported EC effects when the CSs were rated post-pairing than when participants rated novel stimuli post-pairing, both of the present experiments did not find a difference in the size of the EC effect between the two conditions (with Bayesian analysis suggesting moderate evidence for a lack of a difference between the two conditions, even with a prior that assumes a small effect size). Furthermore, a comparison of the post-CS-only EC effects in both conditions with the post-pairing EC effect, suggested that both conditions led to a significant extinction effect. ${ }^{10}$ These results suggest that

${ }^{10}$ Gawronski et al. (2015) did not report a comparison of the post-CS-only EC effects in both conditions with the post-pairing EC effect. However, the corresponding author confirmed in a personal 
the extinction of (the self-reported) EC effects occurs whenever evaluative judgment is made pre-CS-only (post-pairing), though not necessarily evaluative judgment of the CS.

According to the memory-based perspective (e.g., Aust et al., 2019; see also Lipp \& Purkis, 2006), when participants are asked to evaluate the CS both post-pairing and post-CSonly, this repetition changes the strategy they use in their evaluation. Instead of basing their judgment on the integration of all the available evaluative information they encoded about the CS during the experiment, they base their judgment on the evaluative information that they received after their previous rating. The results of the present research might suggest that the rating of novel stimuli and not only the rating of CSs can lead to a context change that in turn leads to a change in the judgment strategy. The possible enhanced demarcation between the pairing and CS-only phases, as a result of the rating task might depend on the similarity between the two rating tasks. If the ratings seem similar, it is easier to think about the whole procedure as a sequence comprised of two pairs of information-judgment (i.e., information, judgment, new information, new judgment). In that respect, it might be essential that the rated stimuli postpairing would be quite similar to the CSs, as in the present experiments. Future research could test the boundary conditions of this effect by using novel stimuli that are less similar to the CSs.

Recently, inhibitory learning was suggested as a mechanism for the extinction effect of EC (e.g., Mierop, Molet, \& Corneille, 2019; Nishiyama, 2020). This perspective was adopted from the literature on extinction effects in Classical Conditioning (CC), in which the dominant idea is that extinction effect reflects new learning of an inhibitory CS-US link that counteracts the activation of the conditioned response (e.g., Bouton, 1993; Delamater, 2004). According to

communication that both conditions in their Experiment 3 also led to a significant extinction effect, as in the present research. 
this perspective, while in $\mathrm{CC}$ the new learning occurs directly as a result of the CS-only presentations, in EC, the learning depends on intentional ratings of the CSs. By default, the CSonly presentations do not elicit that intentional response. The evaluative rating post-pairing prompts a voluntary evaluative judgment during CS-only presentations, leading to an extinction effect (Nishiyama, 2020).

To test the inhibitory leaning explanation for the extinction of EC, previous research examined whether evaluative response production (compared to no production of evaluative response) during CS-only presentations leads to extinction effects. However, the evidence was inconsistent: evaluative response production led to extinction effects in some studies (Nishiyama, 2020), but not in others (Mierop, Molet, \& Corneille, 2019). On one hand, the findings of the current research, that any evaluative rating post-pairing leads to an extinction effect, is compatible with the idea that evaluative ratings post-pairing prompt a voluntary evaluative judgment during extinction which leads to an extinction effect. On the other hand, the inhibitory learning perspective does not explain the lack of extinction effect on indirect evaluation measures. If post-pairing evaluative ratings prompt a voluntary evaluative judgment during extinction, which in turn leads to learning of an inhibitory CS-US link, it is unclear why the new learning would not influence indirect evaluation measures.

\section{Limitations}

First, it is important to note that, although the IAT is a more sensitive indirect measure than the EPT, it also has some limitations. For example, the IAT is sensitive to recoding strategies (e.g., Meissner \& Rothermund, 2013) and to the specific categories and items used to represent these categories (e.g., Foroni \& Bel-Bahar, 2010). Furthermore, the IAT allowed measuring the preference between one of the two positive CSs and one of the two negative CSs. 
That slightly decreased the quality of the indirect measure, in comparison to the self-report questionnaire that measured the evaluation of all four CSs. Therefore, although the present IAT results showed a similar pattern to the EPT results in Gawronski et al. (2015), it is important to continue to investigate the sensitivity of indirect evaluation measures to the extinction of EC using other indirect measures like the recoding-free IAT (Rothermund et al., 2009) or the AMP (Payne et al., 2005).

Second, although null effects found in the present research are compatible with the conclusion that the extinction of EC is limited to evaluation measured directly, inference from a lack of an effect is weak. Furthermore, Bayesian analysis for the key comparison for the indication of extinction (Experiment 2; IAT post-pairing versus IAT post-CS-only when participants evaluate the CS post-rating) showed only anecdotal evidence for a null effect when assuming a small effect size, making the inference from the Bayesian analyses non-definite. Therefore, it is important to continue to accumulate evidence regarding the (lack of) sensitivity of indirect evaluation measures to the extinction of EC.

\section{Conclusion}

The present results are compatible with the findings of Gawronski et al. (2015) that extinction effects on EC are limited to evaluation measured directly with self-report ratings. The present research results suggest that extinction of EC occurs on direct evaluation measures whenever an evaluative judgment is made post-pairing, pre-CS-only, not necessarily evaluative judgment of CS. Future research should further clarify the mechanism underlying these effects and their boundary conditions. 


\section{References}

Aust, F., Haaf, J. M., \& Stahl, C. (2019). A memory-based judgment account of expectancyliking dissociations in evaluative conditioning. Journal of Experimental Psychology: Learning, Memory, and Cognition, 45(3), 417-439.

Baeyens, F., Crombez, G., Van den Bergh, O., \& Eelen, P. (1988). Once in contact, always in contact: Evaluative conditioning is resistant to extinction. Advances in Behaviour Research and Therapy, 10, 179-199.

Baeyens, F., Díaz, E., \& Ruiz, G. (2005). Resistance to extinction of human evaluative conditioning using a between-subjects design. Cognition and Emotion, 19(2), 245-268.

Bar-Anan, Y., \& Nosek, B. A. (2014). A comparative investigation of seven indirect attitude measures. Behavior Research Methods, 46(3), 668-688.

Bouton, M. E. (1993). Context, time, and memory retrieval in the interference paradigms of Pavlovian learning. Psychological Bulletin, 114(1), 80-99.

De Houwer, J., Thomas, S., \& Baeyens, F. (2001). Association learning of likes and dislikes: A review of 25 years of research on human evaluative conditioning. Psychological Bulletin, 127(6), 853-869.

Delamater, A. R. (2004). Experimental extinction in Pavlovian conditioning: Behavioural and neuroscience perspectives. The Quarterly Journal of Experimental Psychology B, 57(2), 97-132.

Fazio, R. H., Jackson, J. R., Dunton, B. C., \& Williams, C. J. (1995). Variability in automatic activation as an unobtrusive measure of racial attitudes: A bona fide pipeline?. Journal of Personality and Social Psychology, 69(6), 1013 -1027. 
Foroni, F., \& Bel-Bahar, T. (2010). Picture-IAT versus Word-IAT: level of stimulus representation influences on the IAT. European Journal of Social Psychology, 40(2), $321-337$.

Gawronski, B., \& De Houwer, J. (2014). Implicit measures in social and personality psychology. Handbook of research methods in social and personality psychology, 2, 283310.

Gawronski, B., Gast, A., \& De Houwer, J. (2015). Is evaluative conditioning really resistant to extinction? Evidence for changes in evaluative judgements without changes in evaluative representations. Cognition and Emotion, 29(5), 816-830.

Greenwald, A. G., McGhee, D. E., \& Schwartz, J. L. (1998). Measuring individual differences in implicit cognition: the implicit association test. Journal of Personality and Social Psychology, 74(6), 1464-1480.

Greenwald, A. G., Nosek, B. A., \& Banaji, M. R. (2003). Understanding and using the Implicit Association Test: I. An improved scoring algorithm. Journal of Personality and Social Psychology, 85(2), 197-216

Hermans, D., Crombez, G., Vansteenwegen, D., Baeyens, F., \& Eelen, P. (2002). Expectancy learning and evaluative learning in human classical conditioning: Differential effects of extinction. In S. P. Shohov (Ed.), Advances in Psychology Research (Vol. 12, pp. 17-40). Hauppauge, NY: Nova Science Publishers

Hofmann, W., De Houwer, J., Perugini, M., Baeyens, F., \& Crombez, G. (2010). Evaluative conditioning in humans: a meta-analysis. Psychological Bulletin, 136(3), 390-421.

Lang, P. J., Bradley, M. M., \& Cuthbert, B. N. (2008). International affective picture 
system (IAPS): Technical manual and affective ratings. Gainesville, FL: The Center for Research in Psychophysiology, University of Florida

Lipp, O. V., \& Purkis, H. M. (2006). The effects of assessment type on verbal ratings of conditional stimulus valence and contingency judgments: Implications for the extinction of evaluative learning. Journal of Experimental Psychology: Animal Behavior Processes, 32(4), 431-440.

Martin, D. (2016). IAT: Cleaning and Visualizing Implicit Association Test (IAT) Data. R package version 0.3. Retrieved from https://CRAN.R-project.org/package=IAT

Meissner, F., \& Rothermund, K. (2013). Estimating the contributions of associations and recoding in the Implicit Association Test: The ReAL model for the IAT. Journal of Personality and Social Psychology, 104(1), 45-69.

Mierop, A., Molet, M., \& Corneille, O. (2019). Response production during extinction training is not sufficient for extinction of evaluative conditioning. Cognition and Emotion, 33(6), 1181-1195.

Morey, R. D., Rouder, J. N., Jamil, T., Urbanek, S., Forner, K., \& Ly, A. (2018). Package 'BayesFactor'. Available at https://cran.rproject.org/web/packages/BayesFactor/BayesFactor.pdf

Nishiyama, R. (2020). Evaluation during the extinction procedure causes extinction in evaluative conditioning. Learning and Motivation, 69, 101600.

Nosek, B. A. (2005). Moderators of the relationship between implicit and explicit evaluation. Journal of Experimental Psychology: General, 134(4), 565-584. 
Nosek, B. A., Greenwald, A. G., \& Banaji, M. R. (2005). Understanding and using the Implicit Association Test: II. Method variables and construct validity. Personality and Social Psychology Bulletin, 31(2), 166-180.

Payne, B. K., Cheng, C. M., Govorun, O., \& Stewart, B. D. (2005). An inkblot for attitudes: affect misattribution as implicit measurement. Journal of Personality and Social Psychology, 89(3), 277-293.

Rothermund, K., Teige-Mocigemba, S., Gast, A., \& Wentura, D. (2009). Minimizing the influence of recoding in the implicit association test: The recoding-free implicit association test (IAT-RF). The Quarterly Journal of Experimental Psychology, 62(1), 8498.

Sagarin, B. J., Ambler, J. K., \& Lee, E. M. (2014). An ethical approach to peeking at data. Perspectives on Psychological Science, 9(3), 293-304.

Stahl, C., \& Aust, F. (2018). Evaluative conditioning as memory based judgment. Social Psychological Bulletin, 13, e28589.

Vansteenwegen, D., Francken, G., Vervliet, B., De Clercq, A., \& Eelen, P. (2006). Resistance to extinction in evaluative conditioning. Journal of Experimental Psychology: Animal Behavior Processes, 32 (1), 71-79.

Walther, E., Weil, R., \& Langer, T. (2011). Why do we like the iPhone? The role of evaluative conditioning in attitude formation. Social and Personality Psychology Compass, 5(7), 473-486. 
